LA-UR $\quad-91-1789$

LA-JR--91-1789

DE91 013375

TITLE: A STUDY OF THE IMPACT OF REACTION RATES ON EQUATION OF STATE

AUTHOR(S) Pier K. Tang

submiredo. APS Topical Conference on Shock Compression of Condensed Matter,

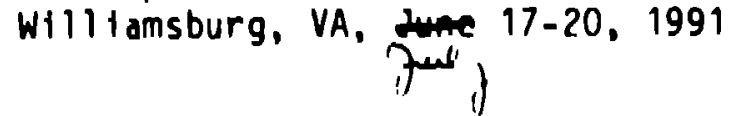

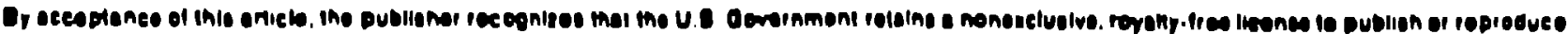

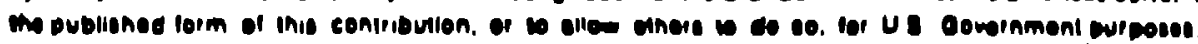

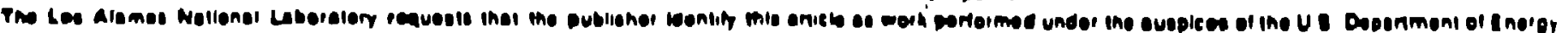

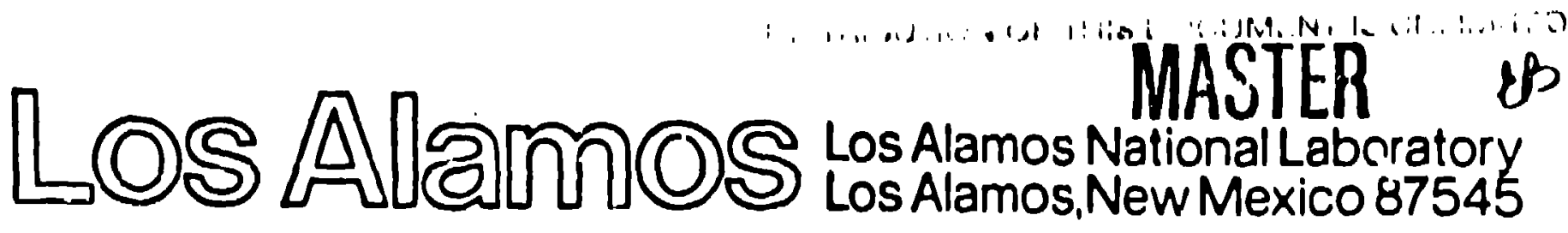




\title{
A §TUDY OF THE IMPACT OF REACTION RATES ON EQUATION OF STATE
}

Pier K. TANG

Los Alamos National Laboratory, Los Alamos, New Mexico 87545

\begin{abstract}
We present experimental evidence on high explosives pushing metal plates that shows the tendency of the equation of state behaving more energetically in the low-pressure region. We believe the deficiency in pre. dicting low- and also high-pressure performance has theoretical origin, and it in related to reaction rate. The reaction process with a slow stage generates a higher effective Chapman-Jou guet pressure. The development of an equation of state based on this information, using the classical detonation theory but without the consideration of the slow component in reaction, would produce a more energetic condition in the low. pressure region and a less powerful one in high pressure. We demonstrate how a polytropic gas equation of state is constructed and the properties it possesses as mentioned. Finally, we show how the effective Chap. man.Jouguet condition comes about and define the property of a pseudo Hugoniot associated with it.
\end{abstract}

\section{INTRODUCTION}

Extending the range of applications beyond the dcmain of calibration is $f$ way of life in engineering and science. In high explosives (HF', we do not usually generate very high pressure in simple experiments, and in other extremes, very low pressure is not easily attainable either. Nev. ertheless, extreme conditions of high and low pressure are often encountered in many applica. tions. To these situations, data developisd for tlie moderate ranges are used.

In previous work through the simulation of plate push experiments, ${ }^{(1)}$ we deacribed how the slow process stage in the reaction can affect the Chapman-Jouguet (CJ) state. It brings about a higher effective pressure than the vornal value based on simple detonation theory. Only in pass. ing did we mention the deficie rey of the EOS in the low-pressure region without giving any rea. on. The product equation of state usod is Beck. or-Kistiakowsky-Wilson (BKW), but the source of the defect is not unique to this particular EOS.

Figire 1 shows the free surface velocity histo. ries of both the experimental and computational resulte for a $50 \cdot \mathrm{mm}$ thick PBX 9602 (95\% TATB. 6\% kol-F B00) puahing alumivum (A) and tenta. lum (Ta) plates of $0.6 \mathrm{~mm}$ thichness. Without the inclumion of alow process, the prediction would fall whort of the experiment in the initial velocity jump, indicating lower C.J presenure condition. ${ }^{(1)}$ With the proper reaction ate, gond match is obtained throughout for Ta; but for $\mathrm{Al}$ the simulation predicts a higinor velocity aner a couple of reverberations. Certainly we could blame the inadequacy of the EOS for Al, but we do have high confideace in the $1,0 S$ uecd because it is obtained from a wide range of experiments. So the fault roust be the HE EOS. To see why this deficiency occurs only when $A$ is used, we examine

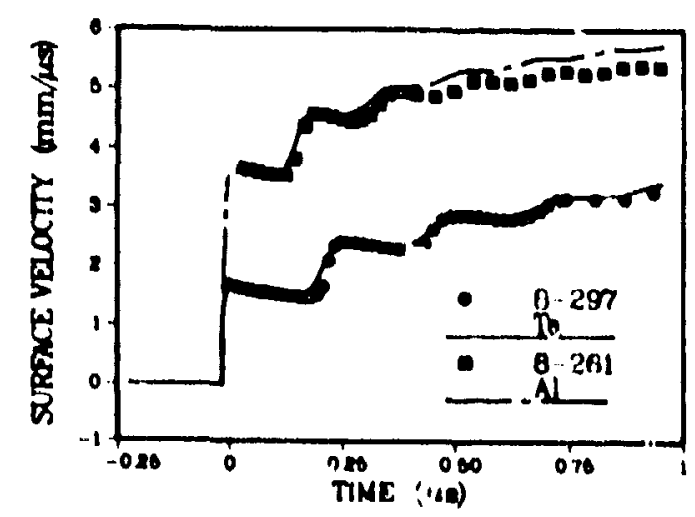

FIOURE I

60-mm PBX 9802 pumhing aluminum and tanen. lum plates. 
the pressure time histories at the explosive-metal interface as seen in Fig. 2. Because of the high impedauce $\mathrm{Ta}$, the pressure is still above $10 \mathrm{GPa}$ around $1 \mu \mathrm{sec}$; but the condition is not so for the low impedance Al. Pressure in the Al case already drops below $5 \mathrm{GPa}$ after the arrival of the second release wave. Similar behavior is seen when a 13 . mm PBX 9502 is used. (1)

Additional evidence is seen in interface velocity experiments, especially for short-duration impact and low-impedance window materials. ${ }^{(2)}$ At this point we begin to suspect the in adequacy of the HE EOS at low pressure, but the question remains. why is it more energetic? Is it merely a condition of defect in numerical extrapolation, or does it have any intrinsic physical significance? This work is to answer the question, if not to offer final solution.

\section{POLYTROPIC GAS EQUATION OF STATE}

To atford our analy'ical investigation, we have to select the simplest equation of state, such as polytropic gas, although we recognize the inadequacy of this particular EOS for condensed phase explosives. However, the most common use of EOS, Jones-Wilkins-Lee (JWL), does have an as. ymptotic behavior of the polytropic gas at low pres. sure. The EOS is ${ }^{(3)}$

$$
p v=(\gamma-1)(e+\lambda q) ;
$$

and the C.I property

$$
p_{1}=D^{2} / v_{0}(\gamma+1) \text {, }
$$

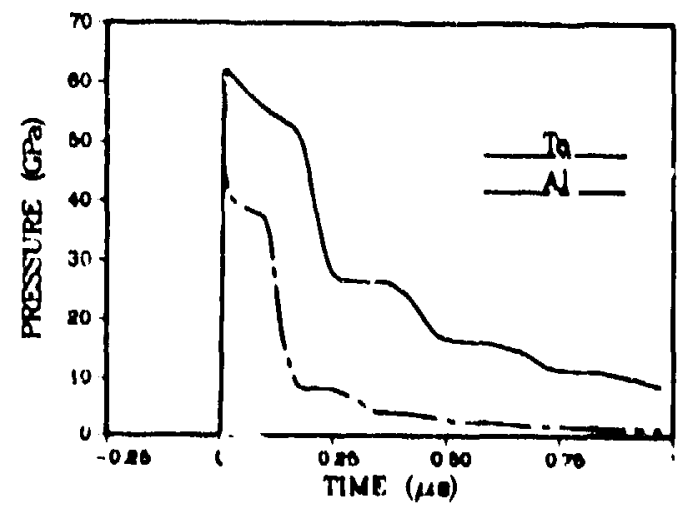

FICURE 2

Pressure histories at interfacen between HE and motnl plates. and

$$
D^{2}=2\left(r^{2}-1\right) q .
$$

The initial pressure is neglected in the formula tion; $p, v$, and $e$ represent pressure, specific vol. ume, and specific internal energy; while $q$ is the leat release, $\gamma$ the polytropic coefficient, and $\lambda$ the reaction fraction. $D$ is the detonation velocity; subscript $c j$ refers to $\mathrm{CJ}$ state, and subscript $o^{\circ} \circ$ initial value. It is a simple matter to construct a polytropic gas EOS if $\mathrm{CJ}$ pressure and detonation velority are known. Choosing dimensionless unit, $v_{0}=1$, and $D^{2}=80$, we have for two different $C . J$ pressures,

$$
\begin{aligned}
& p_{(1)}=32, \gamma=1.50 ; \\
& p_{(1)}=36 ., \gamma=1.22 .
\end{aligned}
$$

The two Hugoniots along with a common Ray. leigh line are shown in Fig. 3 . We see quite clearly that the Hugoniot with higher $C J$ pressure is more energetic than the other in the low-pressure re. gion but less energetic in the high-pressure region, provided they have the same detonation velocity and, therefore, the same Rayleigh line. As we have already noticed in Eq. (1), the form of EOS should show dependence on the reaction fraction $\lambda, 80$ in general.

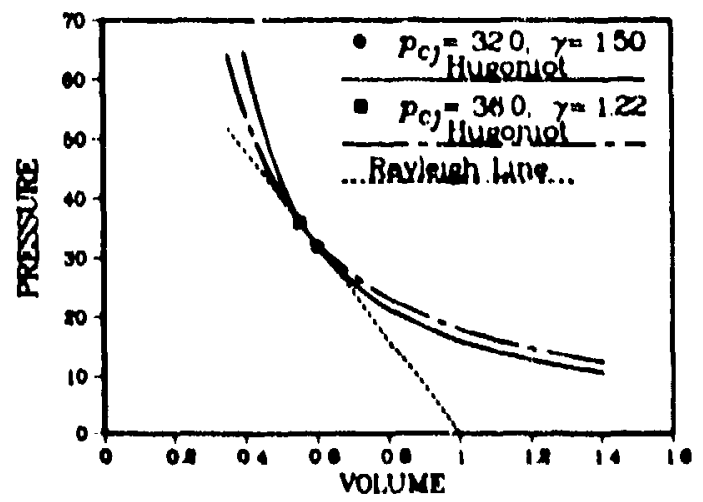

FICIIRE: ?

Construction of a Hugoniot based on ( $S$ atate for a polytropic gas. 


$$
p=p(e, v, \lambda) \text {. }
$$

The appearance of $\lambda$ is critical in expressing the EOS of energetic materials. Only in chemical equilibrium, which assumes infinite rates, can explicit dependence of $p$ on $\lambda$ be removed.

\section{EFFECTTVE CHAPMAN-JOUGUET STATE AND PSEUDO HUGONTOT}

All equations of state require some sort of normalization or parameter adjustment to fit experimental data. In the previous section we have demonstrated such an approach for known detona. tion velocity and $\mathrm{CJ}$ pressure. However, $\mathrm{CJ}$ pressure is not a directly measurable quantity and is inferred only from experiments such as plate push. As we have demonstrated in the simulation of plat a push experiments, a slow component must be added to the reaction process in order to fit the experimentai data better. ${ }^{(1)}$ The consequence of this reaction step is to make the CJ pressure "appear" higher than the normal $C J$ pressure, for the reason given later. Since we can only measure the "effective" CJ pressure, not the normal one, any construction of EOS using the effective value based on classical tetonation theory will lead to wrong re. sult, regardless of the types of EOS.

We determine through interface velocity experi. ments, as well as plate push experiments, that the detonation of condensed high explosives consists of a fast raction atage followed by a slow reaction stage. Figure 4 shows such a reactice history for PBX 9502. The first 85 percent is burned is less than

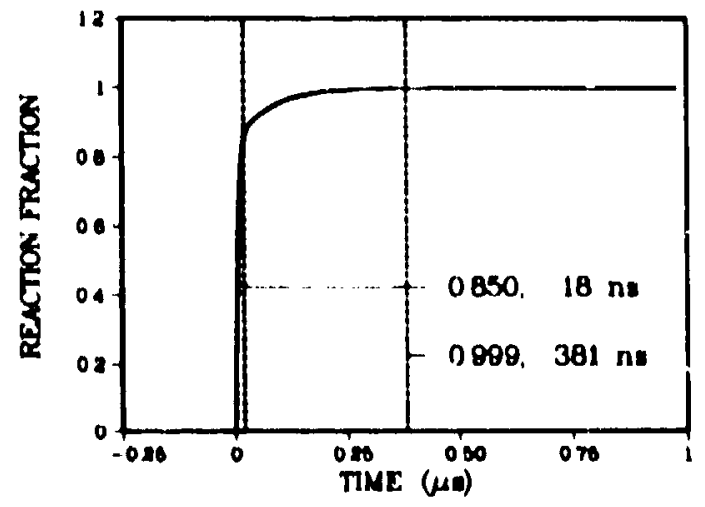

FICURE 4

PBX 9.902 reaction zone showing fart and slow reaction zone thicknesses
$20 \mathrm{~ns}$, but it takes more than $380 \mathrm{~ns}$ to reach the level of 99.9 percent. The question remains why the presence of a slow process would make explosives behave more energetically in the low-pressure regioa as implied through plate push experiments. Referring to Fig. 5, we proceed with the argument. In the $p-v$ plane, first there are two frozen Hugoniots labeled $\lambda=0$ for the initial reactant and $\lambda=1$ for the final product. A Rayleigh line is tan. gent to the product. Hugoniot at the $\mathrm{CJ}$ point; and the intersection of the Raleigh line and the reac. tant Hugoniot is marked VN, the von Neumana spike. If the reaction process is very fast, according to classical steady detonation theory, the state would jump from the initial condition $O$ to $\mathrm{VN}$ and then move down to $\mathrm{CJ}$ along the Rayleigh line. In fact. for an instantaneous reaction as assumed in programmed burn, we do not even concern ourselves with the pathway from VN to $C J$, and the product begins to expand at CJ as soon as the detonation wave arrives. That is why we do not see the VN spike when we use programmed burn.

The second part of our discussion involves a partial!, reacted Hugoniot, labeled $\lambda<1$. This Hugo. nint should be between those of the reactant and the product but much closer to the product oue because of the large amount already reacted as seen in the reaction zone, Fig. 4. The intersection of this Hugosiot and the Rayleigh line is labeled "Effective $\mathrm{C}_{\mathrm{v}}$." Because the first p. se of the reaction is quite fast with a process time of $5 \mathrm{~ns}$, the pathway coming down from $\mathrm{VN}$ follows very

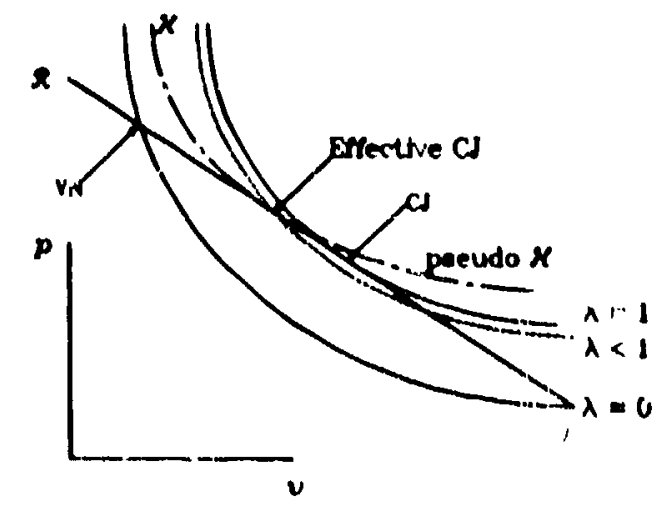

FIOURE?

Evolution of a peoudo Hugoniot baned on an effec. tive C.J ntate. 
closely to the Rayligh line until it reaches the effective $\mathrm{CJ}$ point. The second phase of the reaction is, however, much slower than the first, with a process time of $75 \mathrm{~ns}$. This slow reaction forces the reaction pathway to deviate from the Rayleigh line, starting the expansion from the effective CJ point in a way similar to the classical expansion from the normal CJ state aiong the principal isentrope. The difference is that the composition of the expanding gas through the effect of $\lambda$ is still changing, albeit slowly. From the hydrodynamic point of view, the expansion begins at the effective $C J$ point, not the normal CJ; and, as we can see, the effective CJ pressure is greater than the normal CJ. That is why the inclusion of a slow reaction would result in higher ('J prissure, a condition so vividly illus. trated in plate push experiments. (1)

Let us suppose we have no knowledge of the reaction process - in particular, the slow stage - and proceed to consiruct an EOS based on the measured quautity of " $\mathrm{CJ}$ " pressure. The resulting Hugoniot is shown with the label "pseudo". This Hugoniot satisfies the classical detonation, the tangency, requirement. As long as we do not do anything extraordinary and the form of the EOS is well-behaved, the Hugoniot must have the properties we described earlier for a Hugoniot with high. er C.J pressure, seen in Fig. 3. It appears more energetic than the real Hugoniot in the low. pres. sure region as demonstrated in plate push experiments when Al is used, but less 80 in the high. pressure revion. The same conclusion can be reached about the principal isentrope. This pseudo Hugoniot, unfortunately, forms the basis for the construction of EOS in many cases.

So far we only have direct experimental evi. dence showing the deficiency of the EOS in the low-pressure regime. Still we do not have a concrete result to demonstrate the weakness in the high-pressure domain. However, from the onergy consideration involving carbon congulation, we expect the slow reaction mass fraction should be below 0.1. Instead, value of 0.15 is used. As Figure 2 shows, the $\mathrm{HE}$ experiences a pressure condition over $60 \mathrm{GPh}$, consequence of shock reflection from the Ta plate. The magnitude is twice the val. ue of the quoted normal $C J$ presucure of about 30 GPa. Even reflection from the Al plate can reach a pressure over $40 \mathrm{GPa}$. The scemingly higher value required for the wlow reaction masa fraction is quite likely manifeatation compenanting for the effect of less energy of the BOS in the prensure re. gion above CJ. Some overcompenmation is seen in a
33. nm PHX 9502 pushing Al plate as a result of a slightly larger slow reaction mass fraction. ${ }^{\text {(1) }}$

The above discussion is based entirely on a quasi-steady concept, but in reality, the pathway is much more complex. For one thing, the Rayleigh line is not fixed; it depends on how the system is driven. The effective CJ, shown in Fig. 5, represents a self-supported asymptotic limit. Therefore, the effective $\mathrm{CJ}$ state is not unique with a condi. tion demonstrated clearly in plate push experi. ments showing the change of the initial velocity jump with respect to varying HE thicknass. (3)

\section{CONCLUSIONS}

For a pericd of more than 350 nanoseconds after the onset of detonation, the HE is still in a partial. ly reactud state due to the preseacc of a slow reac. tion. A construction of the EOS without recognizing the fact automatically assumes it to be the complete product and leads to a pselido EOS with the deficiencies described earlier. Because of the finite reaction involved for a rather long peri. od, EOS as presented generically in Eq. (4) has a time-dependent characier through $\lambda$ and consequently results in time-dependent, nonsteady det. onation, a fact that has been observed for some time. To obtain a turue prodact Hugoniut, tue con. dition must be maintained long enough to reach the quasi-steady state uver a wide range of pres. sure conditions. Construction of EOS based in the falso CJ condition alone should bo avoided.

\section{ACKNOWLEDGMENTS}

The author wishes to thank W. Seitz, H. Stacy and J. Wackerle for the experimental data. The wurk is supported by the United States Department of Fin. ergy under Contract W.7405.1\%NG.36.

\section{REFERENCES}

1. P. K. Tang, W. L. Seitz, H. L. Stacy, and J. Wackerle, in Proceedings of Shock Compres. sion of Condensed Matter-1989. Albuquerque. NM, Auguat 14.17, 1988. North-Holland, pp. 278.282.

2. W. I. Seitz, H. L. Stacy, R. Fingelke, P. K. Tang, and J. Wackerle, in Proceedinge of the Ninth Symposium (International) on Detonation, Portiand, OK, August 28. September 1, 1989.

3. W. Fickett and W. C. Davis, Detwation, Uni. veruity of Californin Prem, Berkeley. 1879, pp 18.20. 